\title{
Piecewise Euclidean Structures and Eberlein's Rigidity Theorem in the Singular Case
}

\author{
Michael W Davis \\ BORIS OKUN \\ FANGYANG ZHENG \\ Department of Mathematics, The Ohio State University \\ Columbus, OH 43201, USA \\ Department of Mathematics, Vanderbilt University \\ Nashville, TN 37400, USA \\ Department of Mathematics, The Ohio State University \\ Columbus, OH 43201, USA \\ Email: mdavis@math.ohio-state.edu, okun@math.vanderbilt.edu \\ zheng@math.ohio-state.edu
}

\begin{abstract}
In this article, we generalize Eberlein's Rigidity Theorem to the singular case, namely, one of the spaces is only assumed to be a CAT(0) topological manifold. As a corollary, we get that any compact irreducible but locally reducible locally symmetric space of noncompact type does not admit a nonpositively curved (in the Aleksandrov sense) piecewise Euclidean structure. Any hyperbolic manifold, on the other hand, does admit such a structure.
\end{abstract}

AMS Classification numbers Primary: 57S30

Secondary: $53 \mathrm{C} 20$

Keywords: Piecewise Euclidean structure, CAT(0) space, Hadamard space, rigidity theorem

Proposed: Steve Ferry

Seconded: Walter Neumann, David Gabai
Received: 19 December 1998

Accepted: 27 August 1999

Copyright Geometry and Topology 


\section{Introduction}

Suppose that $V$ and $V^{*}$ are compact locally symmetric manifolds with fundamental groups $\Gamma$ and $\Gamma^{*}$ and with universal covers $X$ and $X^{*}$, respectively. Suppose further that the symmetric spaces $X$ and $X^{*}$ have no compact or Euclidean factors and that no finite cover of $V^{*}$ splits off a hyperbolic 2-manifold as a direct factor. Let $\theta: \Gamma \rightarrow \Gamma^{*}$ be an isomorphism. The Mostow Rigidity Theorem asserts that the isomorphism $\theta$ is induced by an isometry $V \rightarrow V^{*}$, possibly after renormalizing the metric on $V^{*}$. ("Renormalizing the metric" means that we are allowed to rescale the metric on each factor of $X^{*}$ by multiplying by a positive constant.)

In the early eighties, Eberlein, Gromov and Heintze independently proved generalizations of Mostow's Theorem in which the hypotheses on $V$ are weakened. They showed that the conclusion of Mostow's Theorem remains valid without the assumption that $V$ is locally symmetric. It is only necessary to assume that it is a closed Riemannian manifold of nonpositive sectional curvature, provided that the rank of $X^{*}$ is at least two. (If the rank of $X^{*}$ is one, there is no such generalization. For, if $X^{*}$ is of rank one, then the sectional curvature of $V^{*}$ is strictly negative and hence, the same will be true for any sufficiently small deformation of its metric; moreover, the new metric will not, in general, be isometric to the original one.)

Eberlein proved the above generalization of the Rigidity Theorem in the case where $X^{*}$ is reducible and the uniform lattice $\Gamma^{*}$ is irreducible. We shall sometimes refer to this as "Eberlein's case". Gromov proved the result in full generality; however, the argument has only appeared (in [2]) in the case where $X^{*}$ is irreducible.

In this paper we will prove a further generalization of Eberlein's case of the Rigidity Theorem by allowing a further weakening of the hypotheses on $V$ : the metric need not be smooth. To explain this, we first need to understand what "nonpositive curvature" means in this context.

About fifty years ago Aleksandrov showed that the notions of upper or lower curvature bounds make sense for a more general class of metric spaces than Riemannian manifolds, namely, they make sense for "geodesic spaces". A geodesic segment in a metric space $Y$ is the image of an isometric embedding of an interval into $Y$; the space $Y$ is a geodesic space if any two points can be connected by a geodesic segment. A triangle in $Y$ is a configuration of three points (the vertices) and three geodesic segments (the edges) connecting them. Following Aleksandrov, one defines $Y$ to be nonpositively curved if the distance between 
any two points in any small triangle in $Y$ is no greater than the distance between the corresponding points of a comparison triangle in the Euclidean plane. (The precise definition will be given in Section 1.1, below.)

The weakened hypotheses on $V$ will then be that it is a complete geodesic space, that it is nonpositively curved in the above sense and that it is a closed topological manifold.

Our interest in generalizing the Rigidity Theorem to the singular case arose from an attempt to answer the following basic question concerning nonpositively curved, polyhedral metrics on manifolds. Given a Riemannian manifold $M$ of nonpositive sectional curvature, is it possible to find a piecewise Euclidean, polyhedral metric on $M$ which is nonpositively curved in Aleksandrov's sense? An application of our rigidity result is that this is, in fact, impossible when $M=V^{*}$. Indeed, if $V$ were a nonpositively curved polyhedron homeomorphic to $V^{*}$, then by the Rigidity Theorem it would be isometric to $V^{*}$, which is clearly impossible (since $V$ contains open subsets which are flat and $V^{*}$ does not). A further discussion of the problem of approximating nonpositively curved Riemannian metrics by nonpositively curved polyhedral ones will be given in Section 1.

Next we shall make a few remarks concerning the proofs of Mostow and Eberlein. Since $V$ and $V^{*}$ are Eilenberg-MacLane spaces, the isomorphism $\theta: \Gamma \rightarrow$ $\Gamma^{*}$ gives rise to a homotopy equivalence $\bar{f}: V \rightarrow V^{*}$. This lifts to a $\theta-$ equivariant map $f: X \rightarrow X^{*}$. By analyzing the maximal flats in $X$ and $X^{*}$, Mostow first shows that $f$ extends to a homeomorphism of the maximal (or Furstenberg) boundaries. (The maximal boundary of $X^{*}$ is a homogeneous space of the form $G / P$, where $G$ is the identity component of the isometry group $I\left(X^{*}\right)$ and $P$ is a minimal parabolic subgroup.) Let $X^{*}=X_{1}^{*} \times \cdots \times X_{k}^{*}$ be the de Rham decomposition into irreducible factors so that $I\left(X^{*}\right)=I\left(X_{1}^{*}\right) \times \cdots \times I\left(X_{k}^{*}\right)$. The proof of Mostow's Theorem is the easiest to finish in Eberlein's case (where $X^{*}$ is reducible and $\Gamma^{*}$ is irreducible). The key ingredient is the Borel Density Theorem (cf [22]). It implies that the image of $\Gamma^{*}$ is dense in the identity component of each $I\left(X_{i}^{*}\right)$. It follows that the map of maximal boundaries is actually induced by an isomorphism of Lie groups $I_{0}(X) \rightarrow I_{0}\left(X^{*}\right)$ (where $I_{0}$ denotes the identity component of the isometry group). From this, the result follows (cf Corollary 18.2, page 133, in [19]). (A historical remark: one of the earliest rigidity results was proved by Selberg in 1957. He considered the case where $X^{*}$ is the product of $k>1$ copies of the hyperbolic plane and where $\Gamma^{*}$ is irreducible. He used the fact that $\Gamma^{*}$ was dense in each copy of $I_{0}\left(\mathbb{H}^{2}\right)=\operatorname{PSL}(2, \mathbb{R})$ to show that any deformation of $\Gamma^{*}$ in $I\left(X^{*}\right)$ had to be trivial. The details of this argument can be found in [23].) 
There are two steps in Eberlein's generalization of Mostow's argument (to the case where $X$ is not required to be symmetric). First one shows that $X$ also splits isometrically as $X=X_{1} \times \cdots \times X_{k}$. (This is accomplished in [9].) In the second step (which was actually accomplished in the earlier papers [4] and [10]) one shows that each factor $I_{0}\left(X_{i}\right)$ is actually a simple Lie group and that each $X_{i}$ is a symmetric space. Hence, one is reduced back to Mostow's Theorem. The arguments in the second step are mainly Lie group theoretic. The key ingredient is again a density property (which is closely connected to the Borel Density Theorem), namely, that $\Gamma$ satisfies the "duality condition" of [4]. One shows that the the image of $\Gamma$ in $I\left(X_{i}\right)$ is not discrete and hence, that the identity component $G_{i}$ of the closure of its image in $I\left(X_{i}\right)$ is a nontrivial Lie group. One then argues that each $G_{i}$ must be semisimple since otherwise $\Gamma$ (and hence $\Gamma^{*}$ ) would contain a nontrivial normal abelian subgroup.

Our argument, in the case where $X$ is no longer assumed to be Riemannian, follows the same general outline. We show, in Section 2.1, that Eberlein's arguments can be modified to prove that $X$ splits isometrically, as before. As for the second step, we first must face the fact that we don't know, a priori, that the isometry groups $I\left(X_{i}\right)$ are Lie groups. (The Hilbert-Smith Conjecture is still open - if a topological group acts effectively on a topological manifold it is not known if this forces it to be a Lie group.) In fact, a priori, the spaces $X_{i}$, which are manifold factors, need not be manifolds. We get around this by showing, in Section 2.3, that $I\left(X_{i}\right)$ acts transitively on $X_{i}$. (This comes from the fact that $\Gamma$ is dense in $I_{0}\left(X_{i}\right)$.) It then follows from a theorem of Montgomery and Zippin that $I\left(X_{i}\right)$ is a Lie group. Then, following the line of argument in [4] and [10] one can conclude that each $I_{0}\left(X_{i}\right)$ is a simple Lie group. At this stage we are still not done. Even though $X$ is now a homogeneous space of the form $G / K$, the possibility remains that its $G$-invariant metric is not Riemannian. However, we show in Lemma 18 that the nonpositivity of the curvature excludes this possibility.

After writing a preliminary version of this paper we learned of B Leeb's recent preprint [17]. A corollary of his theorem is that the Rigidity Theorem also holds in the singular case whenever each factor of $X^{*}$ has rank $\geq 2$. Leeb's approach generalizes Gromov's line of argument in [2]. Thus, the singular version of the Rigidity Theorem holds in complete generality. We should also mention that Bruce Kleiner can prove our result by a somewhat different argument.

Davis and Zheng were partially supported by NSF grants, Zheng was also partially supported by an Alfred P Sloan Fellowship. This project is also sponsored by the National Security Agency under grant \# MDA 904-98-1-0036.

Geometry and Topology, Volume 3 (1999) 


\section{Piecewise Euclidean CAT(0) structures}

\section{$1.1 \quad$ Hadamard spaces}

A metric space is a length space if the distance between any two points is the infimum of the lengths of all paths between them. The space is a geodesic space if the infimum is always realized by a path of minimum length.

Given any triangle $\Delta$ in a geodesic space $X$ there is a triangle $\Delta^{*}$ in the Euclidean plane with the same edge lengths and a well defined isometry $\Delta \rightarrow \Delta^{*}$ which is denoted by $x \rightarrow x^{*}$. The CAT(0)-inequality of [14] asserts that for any two points $x, y \in \Delta, d(x, y) \leq\left|x^{*}-y^{*}\right|$. (Thus, any triangle in $X$ is "thinner" than the corresponding triangle in $\left.\mathbb{R}^{2}\right)$.

A complete geodesic space $X$ is a Hadamard space (or a $C A T(0)$ space) if each triangle satisfies the $\mathrm{CAT}(0)$-inequality.

A geodesic space is nonpositively curved, if the CAT(0)-inequality holds locally. It can be proved ( $\mathrm{cf}[1]$ or [3]) that if a geodesic space is complete and nonpositively curved, then its universal cover is a Hadamard space.

Example 1 (Aleksandrov and Toponogov) A complete, simply connected Riemannian manifold is a Hadamard space if and only if its sectional curvature is $\leq 0$.

A key fact is that, for any Hadamard space $X$, the metric $d: X \times X \rightarrow \mathbb{R}$ is a convex function, $\mathrm{cf}[14]$. (This means that its restriction to any geodesic segment in $X \times X$ is a convex function.) It follows that any Hadamard space is contractible. (There is an unique geodesic segment from a given base point to any other point; one can then contract the space to the base point by shrinking the geodesic segments.)

A geodesic space has extendible geodesics if every geodesic segment can be extended to a larger interval. A geodesic space is geodesically complete if every geodesic segment can be extended to a geodesic line. An easy argument shows that if a complete geodesic space has extendible geodesics then it is geodesically complete.

Suppose that the geodesic space $X$ is nonpositively curved. If $X$ is a topological manifold (or even if its local homology $H_{*}(X, X-x)$ is nontrivial at each point $x \in X)$, then it has extendible geodesics. Indeed, suppose $c:[0, d] \rightarrow X$ is a geodesic segment which does not extend past $c(0)$. Choose a small ball $B_{\epsilon}$ 
centered at $c(0)$ with $\epsilon<d$ and small enough so that $B_{\epsilon}$ is convex. Since every point in $B_{\epsilon}-c(0)$ can be joined by a geodesic segment to $c(\epsilon), B_{\epsilon}-c(0)$ contracts to $c(\epsilon)$, contradicting the assumption that $H_{*}\left(B_{\epsilon}, B_{\epsilon}-c(0)\right)$ is nonzero.

Given two subsets $A$ and $B$ of $X$, the Hausdorff distance between them, denoted by $H d(A, B)$, is the smallest number $\epsilon$ such that each subset is contained within an $\epsilon$-neighborhood of the other. The subsets $A$ and $B$ are parallel if their Hausdorff distance is finite. An $r$-flat in $X$ is a subset which is isometric to $r$-dimensional Euclidean space.

A subset $A$ of a geodesic space is locally convex if the geodesic segment between any two sufficiently close points in $A$ actually lies in $A$. The subset $A$ is totally geodesic if any geodesic line which contains two distinct points of $A$ is contained in $A$.

Given a closed convex subset $A$ in a Hadamard space $X$, there is a continuous projection map $p: X \rightarrow A$ which sends a point $x \in X$ to the nearest point in $A$.

If the Hadamard space $X$ is locally compact, then it can be compactified by adding an "ideal boundary" $X(\infty)$. One way to define this is as the set of all geodesic rays emanating from a given basepoint. If $X$ is Riemannian, then $X(\infty)$ is homeomorphic to a sphere, but in the general case it can be much more complicated.

Let $\gamma$ be an isometry of $X$. Its translation distance $d(\gamma)$ is the infimum over all points $x \in X$ of $d(x, \gamma x)$. The isometry $\gamma$ is semisimple if there is at least one point $x$ such that $d(\gamma)=d(x, \gamma x) ; \gamma$ is a Clifford translation if $d(\gamma)=d(x, \gamma x)$ for all $x \in X$. If $\gamma$ is semisimple, then its minimum set $M I N(\gamma)$ is the set of $x$ in $X$ such that $d(\gamma)=d(x, \gamma x)$. When $X$ is Hadamard, it follows from the convexity of the metric that $M I N(\gamma)$ is a convex subset.

A length space is singular if it is not isometric to a Riemannian manifold. An important class of singular metrics is the class of polyhedral metrics, defined below.

Suppose that $K$ is a cell complex formed by gluing together convex polytopes in some Euclidean space via isometries between certain faces. Then $K$ is said to have a piecewise Euclidean structure. For example, if the cell complex $K$ is a piecewise linearly embedded subcomplex of Euclidean space (ie, if it is a cell complex in the classical sense), then it has such a structure. A piecewise Euclidean structure on $K$ defines a length metric: the distance between two points is the infimum of the lengths of all piecewise linear paths between them. Such metrics are called piecewise Euclidean (or polyhedral). 
For the further details concerning the definitions and basic properties of $\mathrm{CAT}(0)$ spaces, we refer the reader to the excellent books of Bridson-Haefliger [3] and Ballmann [1].

\subsection{Convex hypersurfaces}

It follows from the Gauss equation that a smooth, convex hypersurface in the Euclidean space has sectional curvature $\geq 0$. The singular analogue of this is the following. The boundary $K$ of a convex polytope in Euclidean space has a piecewise Euclidean metric and this metric is nonnegatively curved in the sense that the reverse inequality to the $\mathrm{CAT}(0)$-inequality is satisfied. In fact, it was precisely for the purpose of studying polyhedral metrics on convex polyhedral surfaces that Aleksandrov initiated the study of length spaces with curvature bounded from below.

The Gauss equation remains valid for the hypersurfaces in manifolds equipped with an indefinite metric, the only change that is necessary is that one side of the equation must be multiplied by -1 when the inner product is negative definite in the normal direction. As we shall see, this sign change allows the possibility that a convex hypersurface in Minkowski space will be nonpositively curved.

Recall that Minkowski space $\mathbb{R}^{n, 1}$ is an $(n+1)$-dimensional real vector space with coordinates $\left(x_{0}, x_{1}, \ldots, x_{n}\right)$ equipped with a Lorentzian inner product of signature $(n, 1)\left(\mathrm{eg}, x \cdot x=-x_{0}^{2}+x_{1}^{2}+\cdots+x_{n}^{2}\right)$. A hyperplane in $\mathbb{R}^{n, 1}$ is spacelike if its normal vector $n$ is timelike, ie, if $n \cdot n<0$. A smooth hypersurface in $\mathbb{R}^{n, 1}$ is spacelike if its tangent space at each point is spacelike. Since the restriction of the inner product to any spacelike hyperplane is positive definite, any spacelike hypersurface inherits a Riemannian structure. By the Gauss equation any smooth, spacelike convex hypersurface in $\mathbb{R}^{n, 1}$ is nonpositively curved.

By definition a convex polyhedral hypersurface $\Sigma$ in $\mathbb{R}^{n, 1}$ is the boundary of an $(n+1)$-dimensional convex polyhedral set $C$ in $\mathbb{R}^{n, 1}$ (ie, $C$ is defined by a discrete set of affine inequalities). The convex hypersurface $\Sigma$ is spacelike if each supporting hyperplane of $C$ is spacelike. As in the Euclidean case, $\Sigma$ has an induced piecewise Euclidean metric.

Example 2 [7] Any spacelike convex polyhedral hypersurface in $\mathbb{R}^{n, 1}$ is a Hadamard space. (Recently, Moussong has extended this result to arbitrary spacelike convex hypersurfaces in $\mathbb{R}^{n, 1}$.)

Geometry and Topology, Volume 3 (1999) 
As a simple corollary we get the following example.

Example 3 [7] Every complete hyperbolic manifold $M^{n}$ admits a nonpositively curved, piecewise Euclidean metric. The construction goes as follows. Identify $M^{n}$ with $\mathbb{H}^{n} / \Gamma$ where $\Gamma$ is a discrete torsion-free subgroup of $O_{0}(n, 1)$, the isometry group of $\mathbb{H}^{n}$. Choose a net $S$ in $M^{n}$ (ie, $S$ is a discrete subset of $M^{n}$ such that each point of $M$ lies within a bounded distance of $S$ ) and let $\widetilde{S}$ denote the inverse image of $S$ in $\mathbb{H}^{n}$. Let $\Sigma$ denote the boundary of the convex hull of $\widetilde{S}$ in $\mathbb{R}^{n, 1}$. Then $\Sigma$ is a $\Gamma$-stable, spacelike, convex polyhedral hypersurface in $\mathbb{R}^{n, 1}$. Hence, as in Example 2, it is $\operatorname{CAT}(0)$. Thus, $\Sigma / \Gamma$ gives the desired piecewise Euclidean metric on $M^{n}$. (The details of this construction can be found in [7].) Similarly, the product of two hyperbolic manifolds can be given a piecewise Euclidean structure. However, if, for example, $\Gamma$ is an irreducible uniform lattice in $I\left(\mathbb{H}^{2} \times \mathbb{H}^{2}\right)$, then, as we shall see in the next section, the resulting $4-$ manifold is rigid.

Remark Gromov has suggested that the construction in Example 3 can be modified to show that any Riemannian manifold of sufficiently pinched negative sectional curvature can be given a nonpositively curved, piecewise Euclidean metric.

\subsection{Questions and further examples}

At this point we have two classes of examples of nonpositively curved geodesic metrics on manifolds: 1) Riemannian metrics and 2) piecewise Euclidean metrics on polyhedral manifolds. What is the intersection of these two classes? When can one type of metric be deformed (through nonpositively curved metrics) into the other type? Both classes contain the Riemannian manifolds of constant curvature 0 (ie, flat manifolds). Example 3 shows that a complete metric of constant curvature -1 can be deformed to a polyhedral metric of nonpositive curvature. Before continuing the discussion let us break our problem into two questions.

Question A Suppose $M^{n}$ is a nonpositively curved Riemannian manifold. Does $M^{n}$ admit a nonpositively curved piecewise Euclidean metric?

Question B Suppose $M^{n}$ is a nonpositively curved piecewise Euclidean polyhedral manifold. Does $M^{n}$ admit a Riemannian metric of nonpositive sectional curvature? 
In dimension 2 both questions have affirmative answers. In dimension 3 the answers are not known (although it seems likely that the answer is "yes" in both cases). As we shall see in Example 6, below, in dimension $\geq 5$, Question B has a negative answer, while in dimension 4 the answer is unknown. A corollary to the main result of this paper is that, in general, Question A also has a negative answer in dimensions $\geq 4$. As we have seen in Example 3, Question A has a positive answer when $M^{n}$ is a real hyperbolic manifold. The authors speculate that the answer will be negative for the other locally symmetric manifolds, eg for complex and quaternionic hyperbolic manifolds.

Example 4 ([14], [5]) Suppose that $V$ is a manifold with a (possibly singular) length metric, that $W$ is a codimension-two submanifold and that $V_{k}$ is a $k$-fold cyclic branched cover of $V$ along $W$. (One way to insure that such branched cover exist, for any $k$, is to require $V$ and $W$ to be closed and oriented and the image $[W]$ of the orientation class to be zero in $H_{n-2}(V)$.)

Theorem A Let $V, W$ and $V_{k}$ be as the above. For the induced metric on $V_{k}$ to be nonpositively curved it is necessary and sufficient that the following two conditions hold:

(a) $V$ is nonpositively curved, and

(b) $W$ is a locally convex subset of $V$.

Proof The necessity of the conditions (a) and (b) is obvious. As for the sufficiency, suppose $\widetilde{\Delta}$ is a small geodesic triangle in $V_{k}$ and that $\Delta$ denotes its image in $V$. If $\widetilde{\Delta}$ intersects the branch set $W$ in a vertex or an edge or if it is contained in $W$, then condition (a) and (b) insure that the distance between two points of $\widetilde{\Delta}$ is the same as that of the corresponding points of $\Delta$. If the intersection of $\widetilde{\Delta}$ and $W$ is one or more points in the interiors of the edges, then we can subdivide $\widetilde{\Delta}$ and apply a standard argument (cf Lemma 3.3, page 15 of [1]) to again conclude that the $\mathrm{CAT}(0)$-inequality holds.

Next we consider some examples which show that in special cases both questions can have positive answers even when the curvature is not constant.

Example 5 [15] Using the arithmetic theory of quadratic forms, one can construct pairs $(V, W)$ as in Example 4 , where $V$ is a hyperbolic $n$-manifold. Consider the quadratic form, $-\sqrt{2} x_{0}^{2}+x_{1}^{2}+\cdots+x_{n}^{2}$. Let $\Gamma_{n}$ denote the group of isometries of this form with coefficients in the ring of integers of $\mathbb{Q}(\sqrt{2})$. 
Then $\Gamma_{n}$ is a discrete cocompact subgroup of $O(n, 1)$. If $\Gamma$ is any torsionfree subgroup of finite index in $\Gamma_{n}$, then $V=\mathbb{H}^{n} / \Gamma$ is a closed hyperbolic $n$-manifold. Note that for each $i, 1 \leq i \leq n$, the hyperbolic reflection $r_{i}$, defined by $\left(x_{0}, \ldots, x_{i}, \ldots, x_{n}\right) \rightarrow\left(x_{0}, \ldots,-x_{i}, \ldots, x_{n}\right)$ lies in $\Gamma_{n}$. If $\Gamma$ is required to be normal in $\Gamma_{n}$, then $r_{i}$ descends to an isometric involution $\overline{r_{i}}$ of $V$. Its fixed point set $V^{\overline{r_{i}}}$ is then a locally convex, codimension-one submanifold of $V$. Consequently, $W=V^{\overline{r_{1}}} \cap V^{\overline{r_{2}}}$ is a locally convex, codimension-two submanifold of $V$. If we further require $\Gamma$ to lie in the identity component of $O(n, 1)$, then both $V$ and $W$ will be orientable. Finally, by passing to a subgroup of index two if necessary, we may suppose, that each $V^{\overline{r_{i}}}$ separates $V$ into two components. This implies that $0=[W] \in H_{n-2}(V)$. Hence, we can obtain a pair $(V, W)$ satisfying all the conditions of Theorem A. It follows that the induced (singular) metric on $V_{k}$ is nonpositively curved. This metric is naturally piecewise hyperbolic. In [15] Gromov and Thurston show that this metric on $V_{k}$ can be smoothed to a Riemannian metric with pinched negative curvature. Moreover, if $n \geq 4, V_{k}$ is not diffeomorphic to a hyperbolic $n$-manifold for infinitely many $k$.

The construction of Example 3 can be used to show that each Gromov-Thurston example also admits nonpositively curved, piecewise Euclidean metric. Simply choose the net $S$ in $V$ so that its inverse image $\widetilde{S}$ in $\mathbb{H}^{n}$ is $\Gamma_{n}$-stable. The convex hull construction then gives a piecewise Euclidean metric on $V$ with the property that each $\overline{r_{i}}$ acts isometrically. Hence, the fixed point sets $V^{\overline{r_{1}}}$ and $V^{\overline{r 2}}$ as well as their intersection $W$ will be locally convex. The induced length metric on $V_{k}$ is piecewise Euclidean and by Theorem A, it is nonpositively curved.

Example 6 [8, page 383] In each dimension $\geq 5$ one can construct a nonpositively curved, piecewise Euclidean metric on a smoothable manifold $M^{n}$ such that its universal cover $X$ (a Hadamard space) is not simply connected at infinity and hence, not homeomorphic to $\mathbb{R}^{n}$. On the other hand, by the Cartan-Hadamard Theorem, if $M^{n}$ admitted a nonpositively curved Riemannian metric, then its universal cover would be homeomorphic to $\mathbb{R}^{n}$. Thus, $M^{n}$ admits no such metric.

\section{Eberlein's Rigidity Theorem in the singular case}

Throughout this section, we shall make the following assumptions. 
Let $\left(X^{*}, d^{*}\right)$ be a reducible global Riemannian symmetric space with no compact or Euclidean factors and of rank $r \geq 2$, and let $\Gamma^{*}$ be an irreducible torsion-free uniform lattice on $X^{*}$. Set $V^{*}=X^{*} / \Gamma^{*}$.

Let $(X, d)$ be a Hadamard space and let $\Gamma$ be a discrete group of isometries on $X$ such that $\Gamma$ and $\Gamma^{*}$ are isomorphic as groups and such that $V=X / \Gamma$ is compact. We further assume that $V$ is a topological manifold.

The goal of this section is to show that the rigidity holds even without the smoothness assumption, namely, one has the following.

Theorem B $(X, d)$ is isometric to $\left(X^{*}, d^{* *}\right)$ for some $d^{* *}$ which differs from $d^{*}$ only by constant multiples on the factors of $X^{*}$.

By the singular analogue ([3], Chapter IV, Theorem 6.1) of the Schroeder's Splitting Theorem, we know that the theorem holds true if $V^{*}$ is (the finite undercover of) the product of several such irreducible compact locally symmetric spaces.

The proof will be a modification of the one given by Eberlein ([9], [10], [4]).

The key to understanding the geometry of a symmetric space $X^{*}$ (reducible or not) is the study of its maximal flats. Given a maximal flat $F^{*}$ in $X^{*}$ and a point $x^{*} \in F^{*}$, one considers the other maximal flats which contain $x^{*}$. It turns out that $F^{*}$ intersects these other maximal flats in a union of hyperplanes. These hyperplanes cut $F^{*}$ into simplicial cones, any one of which is called a Weyl chamber. Given a Weyl chamber and some other point $y^{*} \in X^{*}$, there is a unique maximal flat $F^{*}$ which contains $y^{*}$ and a Weyl chamber parallel to the given one.

A geodesic line in $X^{*}$ is singular if it is contained in more than one maximal flat. It is maximally singular if it is the intersection of the maximal flats which contain it, in other words, if it is an extremal line in a decomposition of a maximal flat into simplicial cones.

\subsection{The image flats}

In this subsection, we don't need the assumption that $X^{*}$ is reducible.

First of all, choose a homotopy equivalence $\bar{f}: V^{*} \rightarrow V$ and an inverse $\bar{g}: V \rightarrow$ $V^{*}$. Let $f$ and $g$ be their lifts to the universal covers. Then $f$ and $g$ are 
$\Gamma$-equivariant, uniformly continuous, and they are $(k, b)$ quasi-isometries, that is,

$$
\frac{1}{k} d^{*}\left(x^{*}, y^{*}\right) \leq d\left(f\left(x^{*}\right), f\left(y^{*}\right)\right) \leq k d^{*}\left(x^{*}, y^{*}\right)
$$

for any $x^{*}, y^{*}$ in $X^{*}$ with $d^{*}\left(x^{*}, y^{*}\right) \geq b$, and similarly for $g$. We may also choose a large constant $A$ such that for any $x^{*}$ and $y^{*}$ in $X^{*}$,

$$
d^{*}\left(x^{*}, g f\left(x^{*}\right)\right) \leq A, \text { and } d\left(f\left(x^{*}\right), f\left(y^{*}\right)\right) \leq k d^{*}\left(x^{*}, y^{*}\right)+A
$$

and similarly for any $x$ and $y$ in $X$,

$$
d(x, f g(x)) \leq A, \text { and } d^{*}(f(x), f(y)) \leq k d(x, y)+A .
$$

The group $\Gamma$ acts on $X^{*}$ via the isomorphism $\Gamma \cong \Gamma^{*}$. An $r$-flat $F^{*} \subseteq X^{*}$ is called $\Gamma$-compact if $\Gamma F^{*} / \Gamma$ is compact. This is equivalent to the condition that $\Gamma$ contains an abelian subgroup $L$ of rank $r$ such that $F^{*}$ is contained in the minimum set of $L$ (which is defined as the intersection of the minimum sets of all $\gamma^{*}$ in $L$ ).

Since $V$ is compact, all elements of $\Gamma$ are semisimple on $X$. So $L$ is an abelian group of semisimple isometries on $X$, therefore in the space $X$, the minimum set $M I N(L)=\mathbb{R}^{r} \times \Sigma \subseteq X$ splits isometrically, with $L$ acting as identity on the convex set $\Sigma$ and as a lattice of translations on $\mathbb{R}^{r}$. Pick any $p \in \Sigma$ and let $F=\mathbb{R}^{r} \times\{p\}$, then it is clear that both $H d\left(F, f\left(F^{*}\right)\right)$ and $H d\left(F^{*}, g(F)\right)$ are finite, since all of the subsets involved are $L$-invariant and have compact quotients. Therefore, we have proved the following:

For any $\Gamma$-compact $r$-flat $F^{*} \subseteq X^{*}$, there exists $r$-flat $F \subseteq X$ such that $H d\left(F, f F^{*}\right)<\infty$.

Note that $H d\left(g F, F^{*}\right)<\infty$, and the $r$-flat $F^{*}$ in $X^{*}$, which satisfies this is unique. So, by the Quasi-flat Theorem of Kleiner-Leeb [16] or Eskin-Farb (Corollary 7.4, [13]), we know that there exists a constant $R^{\prime}>0$, which is independent of the choice of flats, such that $H d\left(g F, F^{*}\right) \leq R^{\prime}$. By letting $R=k R^{\prime}+2 A$, we have the following lemma.

Lemma 1 There exists a constant $R>0$ such that for any $r$-flat $F^{*} \subseteq X^{*}$, there is a $r$-flat $F \subseteq X$ with Hausdorff distance $H d\left(F, f F^{*}\right) \leq R$.

Furthermore, if $F_{1}$ is any $r$-flat in $X$ with $H d\left(F_{1}, f F^{*}\right)<\infty$, then $H d\left(F_{1}, f F^{*}\right) \leq R$.

Proof From the above discussion, the statement holds when $F^{*}$ is $\Gamma$-compact. Since the set of $\Gamma$-compact $r$-flats is dense in the set of all $r$-flats in $X^{*}$, and any sequence of $r$-flats in $X$ which all meet a compact set will have a convergent subsequence, the general case follows. 
We will call such a flat $F$ an image flat of $F^{*}$, and $F^{*}$ the preimage flat of $F$. Note that the preimage flat of $F$ is unique, while union of all the image flats of $F^{*}$ forms a closed convex set which splits as a product $F \times \Sigma$, with $\Sigma$ convex and having diameter $\leq 2 R$.

\section{$2.2 \quad$ The reducibility of $X$}

In this subsection, we want to show that the reducibility result of [9] holds in the singular case as well. Most of the proof from [9] goes through, with the following modifications.

First, we need to establish the so-called "duality condition" of $\Gamma$ on $X$. Recall that $\Gamma$ satisfies the duality condition on $X$, if for any geodesic $c$ in $X$, there exists a sequence $\left\{\gamma_{n}\right\} \subseteq \Gamma$ such that $\gamma_{n}(x) \rightarrow c(+\infty)$ and $\gamma_{n}^{-1}(x) \rightarrow c(-\infty)$. To establish this we need the following lemma.

Lemma 2 Let $c$ be a geodesic in $X$. Then there exists an image flat $F$ of a flat $F^{*} \subseteq X^{*}$, such that $d(c(t), F)$ is constant.

Proof Since there are $r$-flats in $X^{*}$ passing through any two given points, we may choose $F_{n}^{*} \subseteq X^{*}$ passing through $g(c(n))$ and $g(c(-n))$. Let $F_{n}$ be an image flat of $F_{n}^{*}, n=1,2, \ldots$. Since

$$
d\left(c( \pm n), F_{n}\right) \leq A+d\left(f g(c( \pm n)), f\left(F_{n}^{*}\right)\right)+R=A+R
$$

by the convexity of the metric, we have $d\left(c(0), F_{n}\right) \leq A+R$, so by passing to a subsequence, we may assume that $F_{n_{k}} \rightarrow F$ and $F$ is an image flat of $F^{*}$, the limit of the $F_{n_{k}}^{*}$. We have $d\left(c\left( \pm n_{k}\right), F\right) \leq A+R$ is bounded for all $k$; hence, $d(c(t), F)$ is constant, by the convexity of the metric.

Lemma 3 Any finite index subgroup of $\Gamma$ satisfies the duality condition on $X$.

Proof Fix a geodesic $c$ in $X$. By Lemma 2, there exists a flat $F^{*}$ and an image flat $F$ such that $c$ has constant distance from $F$. Let $F_{n}^{*}$ be a sequence of $\Gamma$-compact flats which converges to $F^{*}$. For each n, let $F_{n}$ be an image flat of $F_{n}^{*}$, and denote by $L_{n} \subseteq \Gamma$ the corresponding lattice on $F_{n}^{*}$ or $F_{n}$. Passing to subsequence if necessary, we may assume that $F_{n} \rightarrow \widetilde{F}$, which is also an image flat of $F^{*}$, hence $c$ has constant distance to $\widetilde{F}$. Let $\tilde{c}$ be the projection of $c$ onto $\widetilde{F}$. We can choose a sequence of $L_{n}$-compact geodesics $c_{n} \subseteq F_{n}$ such 
that $c_{n} \rightarrow \tilde{c}$. That is, there exists a sequence $\left\{\gamma_{k}^{(n)}\right\} \subseteq L_{n} \subseteq \Gamma$ such that for each $n, \gamma_{k}^{(n)}\left(c_{n}(0)\right) \rightarrow c_{n}(+\infty)$ and $\left(\gamma_{k}^{(n)}\right)^{-1}\left(c_{n}(0)\right) \rightarrow c_{n}(-\infty)$ when $k \rightarrow \infty$. By taking a diagonal subsequence in $\left\{\gamma_{k}^{(n)}\right\}$, we know that there exists sequence $\left\{\phi_{n}\right\} \subseteq \Gamma$ such that $\phi_{n}(x) \rightarrow c(+\infty)$ and $\phi_{n}^{-1}(x) \rightarrow c(-\infty)$ as $n \rightarrow \infty$.

Lemma 4 (cf Lemma 2.4a of [4]) Let $x \in X(\infty)$ be arbitrary and let $y \in$ $X(\infty)$ be a point that can be joined by a geodesic to $x$. If $z \in X(\infty)$ is any point that can be joined to $x$ then $z \in \overline{\Gamma(y)}$.

Proof With the duality condition established as above, the proof of Lemma $2.4 \mathrm{a}$ in [4] can be easily modified to cover the singular case. All we have to do is to estimate $\measuredangle_{q}\left(\phi_{n} y, \phi_{n} p\right)$ the same way for a point $p$ on $\gamma$ and allow $q$ to be an arbitrary but fixed point in $X$, then $\measuredangle_{q}(w, z)=0$ for any $q$ would imply $w=z$. Here $w$ is any accumulation point of $\phi_{n} y$.

With the duality condition established, let us walk through the proofs in sections 4 and 5 of [9]. Our metric space $(X, d)$ is no longer smooth and we don't have the uniqueness of continuation of geodesic segments. Our starting point is the following proposition.

Proposition 1 (cf Proposition 4.1 of [9]) Let $\gamma^{*}$ be a maximally singular geodesic of $X^{*}$ and let $F^{*}$ be an $r$-flat containing $\gamma^{*}$. Let $F$ be an $r$-flat in $X$ such that $H d\left(f\left(F^{*}\right), F\right)<\infty$. Then

(1) the points $x=\lim _{t \rightarrow+\infty}\left(f \circ \gamma^{*}\right)(t)$ and $y=\lim _{t \rightarrow-\infty}\left(f \circ \gamma^{*}\right)(t)$ exist and are distinct in $X(\infty)$

(2) for any point $p$ in $F$ it follows that $\measuredangle_{p}(x, y)=\pi$ and there exists the unique geodesic $\gamma$ joining $x$ and $y$ passing through $p$, and $\gamma$ is contained in $F$.

Proof For Proposition 1, the proof given in the appendix of [9] works. The only place where care needs to be taken is with regard to the paragraph on page 73 , where the angles are used. In a general Hadamard space, the angle function $\measuredangle_{x}(y, z)$, while it is still continuous in $y$ and $z$, is only upper semicontinuous with respect to the vertex $x$. Also, two different geodesics from $x$ could form a zero angle (in fact they could even share a segment).

Note that given two distinct points $u, v$ in $X(\infty)$, there always exists a point $q \in X$ such that $\measuredangle_{q}(u, v)>0$. Also, $\measuredangle_{q}(u, v)$ is continuous in $u$ or $v$ on $\bar{X}=X \cup X(\infty)$, and satisfies the triangle inequality

$$
\measuredangle_{q}(u, v) \leq \measuredangle_{q}(u, w)+\measuredangle_{q}(w, v) .
$$


With these properties, the paragraph on page 73 of [9] can easily be modified and the proof is valid.

Now we consider the situation where $X^{*}=X_{1}^{*} \times X_{2}^{*}$. Define $S^{*} \subset X^{*}(\infty)$ to be the set of endpoints at infinity of the maximally singular geodesics of $X^{*}$ and let $S_{i}^{*}=S^{*} \cap X_{i}^{*}$ for $i=1,2$. ( $S^{*}$ is the set of maximally singular points in $X^{*}(\infty)$.)

By Proposition 1 the map $f$ defines an equivariant map $\bar{f}: S^{*} \rightarrow X(\infty)$. Let $S_{i}$ denote the closure of $\bar{f}\left(S_{i}^{*}\right)$ in $X(\infty)$.

Proposition 2 (cf Proposition 4.3 of [9]) Let $S_{1}, S_{2}$ be as above. Then:

(1) Each of the sets $S_{1}, S_{2}$ is invariant under $\Gamma$.

(2) A point $x \in S_{i}$ can only be joined by a geodesic to some other point in $S_{i}$.

Proof (1) is obvious. (2) follows by taking closures from the fact that, for $x \in \bar{f}\left(S_{i}^{*}\right)$, the point $y$ of Proposition 1 belongs to $S_{i}$, together with part (1) and Lemma 4.

Now let us continue with the proofs in Appendix 2 of [9]. We start with Lemma 5.1. Lemma 5.1 is needed in the proof of Proposition 5.1 and Sublemma 5.4b. We will state the following weaker version, which is sufficient for our purpose.

Lemma 5 (cf Lemma 5.1 of [9]) Suppose $\gamma(t)$ is a geodesic in the CAT(0) space $X$ and write $x=\gamma(\infty), y=\gamma(-\infty), p=\gamma(0)$. Denote by $L(p, x)$ and $B(p, x)$ the horosphere and horoball, respectively, which are centered at $x$ and pass through the point $p$. Let $C=C_{x y}=\left\{q \in X \mid \measuredangle_{q}(x, y)=\pi\right\}$. Then $L(p, x) \cap L(p, y)=B(p, x) \cap B(p, y), C$ is the union of all the geodesics parallel to $\gamma, C$ is a closed convex subset of $X$, and $C$ splits as $C=\gamma \times C^{\prime}$ for a closed convex subset $C^{\prime} \subseteq L(p, x) \cap L(p, y)$.

Proof In the singular case, it is still true that if two geodesic rays meet at $q$ with angle $\pi$, then they form a geodesic. Also, two parallel geodesics bound a flat strip (the 'Flat Strip Lemma' or 'Sandwich Lemma', see for example [2] or [3]). By the definition of Busemann function (see [2]) and the Law of Cosine in a comparison triangle, it is not hard to see that if $q \in B(p, x)$, then $\measuredangle_{p}(x, q) \leq \frac{\pi}{2}$. Also, if $q \in B(p, x) \backslash L(p, x)$, then $\measuredangle_{p}(x, q)<\frac{\pi}{2}$. From this it follows that $B(p, x) \cap B(p, y) \subseteq L(p, x) \cap L(p, y)$. 
For each point $p \in X$, let $B_{p}$ denote the smallest convex subset of $X$ which contains all the maximally singular geodesics through $p$ with endpoints in $S_{1}$.

Proposition 3 With notation as in Lemma 5, suppose $x \in S_{2}$. Then $B_{p}$ is contained in an $R^{\prime}$-neighborhood of $C_{x y}$, for some constant $R^{\prime}$.

Proof This is essentially the proof of Proposition 5.1 of [9], but since we do not assume that $C_{x y}$ is a proper subset of $X$, we get a weaker result.

Proposition 4 (cf Proposition 5.2 of [9]) If $r \in B_{p}$ then $B_{r} \subseteq B_{p}$.

Proof The proof of Proposition 5.2 of [9] is valid in the singular case as well.

Before stating the next proposition we need the following four lemmas.

Lemma 6 (cf Lemma 5.5a of [9]) Let $p, r$ be any two points of $X$. Then $d\left(q, B_{r}\right) \leq d\left(p, B_{r}\right)$ for every point $q \in B_{p}$. Moreover suppose that $p \notin B_{r}$ and let $P: X \rightarrow B_{r}$ denote the nearest point projection. Then for every $x \in S_{1}$ and any geodesic $\gamma$, with $\gamma(0)=p$ and $\gamma(\infty)=x$, the projection $(P \circ \gamma)(t)$ is a unit speed geodesic and $\gamma$ and $P \circ \gamma$ bound a flat strip in $X$.

Lemma 7 ([1, page 25, Corollary $5.8(\mathrm{i})])$ Let $p, q \in X$ and $x \in X(\infty)$. If $\measuredangle_{p}(x, q)+\measuredangle_{q}(x, p)=\pi$, then the three points span a flat strip.

Lemma 8 (cf Sublemma 5.4b of [9]) Let $\gamma$ be a geodesic of $X$ such that $\gamma[0,+\infty) \subseteq B_{p}$, where $p=\gamma(0)$. Let $z=\gamma(+\infty)$. Then there exists $x \in S_{1}$ and $t \in \mathbb{R}$ such that $\measuredangle_{\gamma(t)}(x, z) \neq \pi / 2$.

Proof The proof of Sublemma 5.4b of [9] holds true using Lemma 5 and Lemma 7, instead of Lemma 5.1 and Sublemma 5.4a of [9].

Lemma 9 (cf Lemma 5.4 of [9]) For every point $p \in X$ there exist a number $A>0$ such that $d\left(p, B_{r}\right)<A$ for every $r \in B_{p}$.

Proof Again, the proof of Lemma 5.4 of [9] works using Lemma 6 instead of Lemma 5.5a of [9] and the fact that the limit of a convergent sequence of (half) flats is still a (half) flat. 
Proposition 5 (cf Proposition 5.4 of [9]) There exists a point $p \in X$ such that $B_{r}=B_{p}$ for every point $r \in B_{p}$.

Proposition 6 (cf Proposition 5.5 of [9]) For every point $p \in X$ and every point $r \in B_{p}, B_{r}=B_{p}$.

Proposition 7 (cf Proposition 5.6 of [9]) For every point $p \in X$ the set $B_{p}$ is complete, totally geodesic subspace of $X$.

Proof The proof of Proposition 5.6 of [9] goes through without change, except for the last paragraph of the proof on page 63, where angles are again used. What is needed to complete the proof is the following lemma.

Lemma 10 Consider a geodesic triangle with vertices $p, q$, and $r$. Let $\sigma(t)$, $t \in[0, d(p, q)]$, be the geodesic segment from $p$ to $q$, with $\sigma(0)=p$. If there is a sequence $\left\{t_{i}\right\}$ of small positive numbers that converges to 0 , such that $d(r, p) \leq d\left(r, \sigma\left(t_{i}\right)\right.$ for each $i$, then $\measuredangle_{p}(q, r) \geq \frac{\pi}{2}$.

Proof Let $\gamma(t)$ be the geodesic segment from $p$ to $r$, with $\gamma(0)=p, \gamma(l)=r$, where $l=d(p, r)$. Denote by $d_{s t}$ the distance between $\gamma(s)$ and $\sigma(t)$. Then the function

$$
f(t, s)=\left(t^{2}+s^{2}-d_{s t}^{2}\right) / 2 s t
$$

is monotonically decreasing in $s>0$ and $t>0$, and it's limit when $s \rightarrow 0$, $t \rightarrow 0$ is the cosine of the angle $\measuredangle_{p}(q, r)$.

Now assume that $\measuredangle_{p}(q, r)<\frac{\pi}{2}$. Then there exist $t>0$ and $s>0$ such that $f(t, s) \geq \epsilon>0$. By the monotonicity of $f$ in $t$, we have

$$
\left(t^{\prime 2}+s^{2}-d_{s t^{\prime}}^{2}\right) / 2 s t^{\prime} \geq \epsilon
$$

for any $0<t^{\prime} \leq t$. So for $t^{\prime}$ sufficiently small, we have $s>d_{s t^{\prime}}$. Hence for $t_{i}$ sufficiently small,

$$
d(r, p)=d(r, \gamma(s))+s>d(r, \gamma(s))+d_{s t_{i}} \geq d\left(r, \sigma\left(t_{i}\right)\right.
$$

which contradicts our assumption.

To complete the proof of Proposition 7, we apply the lemma to the triangle with vertices $q_{1}, \bar{q}$ and $r$. We have that two of the internal angles are at least $\frac{\pi}{2}$. So the sum of the internal angles is at least $\pi$, which implies that it must be a triangle in $\mathbb{R}^{2}$, with two right internal angles. This is clearly impossible. 
Summarizing the above discussions, we can now state the Eberlein's result in [9] in the singular case as the following:

Theorem C Let $X^{*}=X_{1}^{*} \times \cdots \times X_{k}^{*}$ be the decomposition into irreducible factors, $k \geq 2$. For $1 \leq i \leq k$, denote by $S_{i}^{*} \subseteq X_{i}^{*}(\infty)$ the set at infinity of the maximally singular geodesics in $X_{i}^{*}$. Then the following holds:

(1) For any maximally singular geodesic $c^{*} \subseteq X^{*}$, there exists a geodesic $c \subseteq X$ such that the Hausdorff distance $H d\left(c, f\left(c^{*}\right)\right) \leq R_{1}$ for some uniform constant $R_{1}$.

(2) If $c^{*}$ is the intersection of $r$-flats $F_{1}^{*}, \ldots, F_{m}^{*}$, and $c_{1}$ is a geodesic parallel to all the image flats $F_{1}, \ldots, F_{m}$, then $c_{1}$ is parallel to $c$.

(3) Let $S_{i} \subseteq X(\infty)$ be the image of $S_{i}^{*}$ guaranteed by (1). For any $x \in X$, denote by $A_{i}(x)$ the union of all the geodesic rays from $x$ to the points in $S_{i}$, and let $B_{i}(x)$ be the smallest closed convex subset of $X$ that contains $A_{i}(x)$. Then each $B_{i}(x)$ is totally geodesic in $X$, and $B_{i}(x)$ is parallel to $B_{i}(y)$ for any $x, y \in X$.

(4) For any $x \in X$ and $z \in S_{j}$, let $c$ be a geodesic with $c(0)=x$ and $c(\infty)=z$. Then for $i \neq j, B_{i}(x) \subseteq N_{R^{\prime}}\left(P_{c}\right)$, the $R^{\prime}$-neighborhood of $P_{c}$. Here $P_{c}$ denotes the set of points $y \in X$ where there is a geodesic through $y$ that is parallel to $c$.

\subsection{Transitivity of the isometry group on $X$}

Our next goal is to establish the fact that the isometry group $I(X)$ of $X$ acts transitively on $X$, hence $I(X)$ is a Lie group by the theorem of Montgomery and Zippin [20].

Let $X^{*}=X_{1}^{*} \times \cdots \times X_{k}^{*}$ be the decomposition into irreducible factors. By assumption, $k \geq 2$. In order to distinguish the actions of $\Gamma$ on $X^{*}$ and $X$, we will denote the lattice on $X^{*}$ by $\Gamma^{*}$. By passing to a sublattice if necessary, we may assume that the irreducible lattice $\Gamma^{*} \subseteq I_{0}\left(X^{*}\right)=I_{0}\left(X_{1}^{*}\right) \times \cdots \times I_{0}\left(X_{k}^{*}\right)$, where $I_{0}$ stands for the identity component of the isometry group. So each $\gamma^{*} \in \Gamma^{*}$ is in the form $\gamma^{*}=\left(\gamma_{1}^{*}, \ldots, \gamma_{k}^{*}\right)$. Denote by $\Gamma_{i}^{*}$ the projection of $\Gamma^{*}$ on $X_{i}^{*}$. Since $\Gamma^{*}$ is assumed to be irreducible, by Borel Density Theorem, we know that for each $1 \leq i \leq k, \Gamma^{*} \rightarrow \Gamma_{i}^{*}$ is injective, and $\Gamma_{i}^{*}$ is dense in $I_{0}\left(X_{i}^{*}\right)$.

From the discussion in the last subsection, we know that for each $1 \leq i \leq k$, $X$ is foliated by parallel, totally geodesic leaves $B_{i}$. By the Sandwich Lemma, 
for each $i, X$ splits isometrically as $B_{i} \times Y_{i}$. Basically, we want to show that these foliations are mutually perpendicular, and hence, that $X$ has a product structure corresponding to that of $X^{*}$. But we can only prove this in the orthogonal complement of the Euclidean factor of $X$.

Denote by $C(X)$ the set of all Clifford translations in $I(X)$, the isometry group of $X$. It is a normal abelian subgroup of $I(X) . X$ splits isometrically $X=$ $\mathbb{R}^{s} \times Y, s \geq 0$, such that each $\phi \in C(X)$ has the form $(T, i d)$ under this splitting with $T$ a translation, and so that $Y$ does not contain any Euclidean factor. Furthermore, any isometry of $X$ respects this splitting: $I(X)=I\left(\mathbb{R}^{s}\right) \times I(Y)$. $(\operatorname{cf}(6.2)$ of $[2])$.

Our first goal is to show that, for each $1 \leq i \leq k$, the leaves of $B_{i}$ all respect the product structure $X=\mathbb{R}^{s} \times Y$. To be more precise, for any $x=\left(x_{0}, y\right) \in X$, $B_{i}(x)=B_{i 0}\left(x_{0}\right) \times B_{i Y}(y)$, and $B_{i 0}$ (or $\left.B_{i Y}\right)$ is a parallel totally geodesic foliation in $\mathbb{R}^{s}$ (or $Y$ ).

As before, for each $1 \leq i \leq k$, let $S_{i}^{*} \subseteq X_{i}^{*}(\infty)$ denote the set at infinity of the maximal singular geodesics in $X_{i}^{*}(1 \leq i \leq k)$, and $S_{i} \subseteq X(\infty)$ the image guaranteed by part (1) of Theorem C. Again denote by $A_{i}(x)$ the union of all geodesic rays from $x$ to the points in $S_{i}$, and $B_{i}(x)$ the smallest closed convex set containing $A_{i}(x)$.

Now consider an image flat $F \subseteq X$. Let us denote by $F_{0}$ and $F_{Y}$ the projection of $F$ in $\mathbb{R}^{s}$ and $Y$, respectively. If we fix a point $x=\left(x_{0}, y\right) \in F$ and regard $F_{0} \subseteq \mathbb{R}^{s} \times\{y\}, F_{Y} \subseteq\left\{x_{0}\right\} \times Y$, then we have the following lemma.

Lemma 11 For any image flat $F$ in $X, F=F_{0} \times F_{Y}$.

Proof Since any image flat is parallel to an image flat that is the limit of a sequence of $\Gamma$-compact image flats, it suffices to prove the lemma for $\Gamma$-compact image flats. Let $L \subseteq \Gamma$ be a rank $r$ abelian subgroup which acts as a lattice on $F$. For any $\gamma \in L$, write $\gamma=\left(\gamma_{0}, \gamma_{Y}\right)$ under the splitting $X=\mathbb{R}^{s} \times Y$. Then $\gamma_{0}$ is a translation on $F_{0}$. This is true because, for any geodesic $c_{0}$ in $F_{0}, c_{0}$ is the projection of some geodesic $c$ in $F$. Since $\gamma$ maps $c$ to a parallel geodesic in $F, \gamma_{0}\left(c_{0}\right)$ is parallel to $c_{0}$. Let $T$ be a translation in $F_{0}$. As $T$ commutes with $\gamma_{0}$ for all $\gamma \in L, T F$ is also invariant under $L$. Since we have uniform bound in Hausdorff distance between $g(F)$ or $g(T F)$ and $F^{*}$, the preimage flat, we know that the Hausdorff distance between $F$ and $T F$ must be bounded by a uniform constant. Therefore, $F_{0}$ must be contained in $F$, since otherwise we can choose a translation in $F_{0}$ that sends $F$ to a parallel $T F$ with arbitrarily large $H d(F, T F)$.

Geometry and Topology, Volume 3 (1999) 
As a corollary of this, we have the following.

Lemma 12 For each $1 \leq i \leq k$, the set $S_{i} \subseteq \mathbb{R}^{s}(\infty) \cup Y(\infty)$. So the foliation $B_{i}$ is a product foliation $B_{i 0} \times B_{i Y}$. Furthermore, $Y=B_{1 Y} \times \cdots \times B_{k Y}$.

Proof Since any maximally singular geodesic $c^{*} \subseteq X_{i}^{*}$ is an intersection of $r$-flats, there exist $r$-flats $F_{1}^{*}, \ldots, F_{m}^{*}$ with $c^{*}=F_{1}^{*} \cap \ldots \cap F_{m}^{*}$. Let $F_{j}$ be an image flat of $F_{j}^{*}, 1 \leq j \leq m$. Then part (1) of Theorem $\mathrm{C}$ says that there exists a geodesic $c \subseteq X$ which is parallel to all $F_{j}$, and any geodesic $\tilde{c}$ parallel to all $F_{j}$ must be parallel to $c$. In the product space $X=\mathbb{R}^{s} \times Y, c$ is parallel to $F_{j}$ if and only if $c_{0}$ is parallel to $F_{0}$ and $c_{Y}$ is parallel to $F_{Y}$. Here the subscripts denote the projections. So if $c$ is not parallel to a leaf of $\mathbb{R}^{s}$ or $Y$, then any line in the plane $c_{0} \times c_{Y}$ would be parallel to all those $F_{j}$, which is impossible. So, $c$ must be parallel to either $\mathbb{R}^{s}$ or $Y$. That is, $S_{i}$ is contained in the disjoint union of $\mathbb{R}^{s}(\infty)$ and $Y(\infty)$.

From this and the definition of $B_{i}$, it is clear that for each $i, B_{i}=B_{i 0} \times B_{i Y}$ is a product foliation.

Next we claim that for $i \neq j, B_{i Y}$ and $B_{j Y}$ are perpendicular. Take any geodesic $c$ with $c(\infty) \in S_{j} \cap Y(\infty)$. Then any leaf $B_{i}(x)$ is contained in the neighborhood $N_{R^{\prime}}\left(P_{c}\right)$ by part (4) of Theorem C. Here $P_{c}$ stands for the union of all geodesics parallel to $c$. So if the projection $\bar{c}$ of $c$ onto $B_{i Y}$ is not a point, any point in $B_{i Y}$ will be within distance $R^{\prime}$ to another point in $B_{i Y}$ where there is a geodesic parallel to $\bar{c}$ passing through. That is, $B_{i Y} \subseteq N_{R^{\prime}}(P)$, where $P$ is the union of all geodesics in $B_{i Y}$ that is parallel to $\bar{c}$. Since $B_{i Y}$ is geodesically complete, and $P \subseteq B_{i Y}$ is convex, we know that $B_{i Y}=P$. That is, $B_{i Y}$ is foliated by geodesics parallel to $\bar{c}$, so $B_{i Y}$, hence $Y$ will contain a Euclidean factor, a contradiction. So geodesics from $x \in B_{i Y}$ to any point in $S_{j} \cap Y(\infty)$ must be perpendicular to $B_{i Y}$. So $A_{j}(x)$ must be contained in $Z(x)$, the leaf through $x$ of the orthogonal complement of $B_{i Y}$ in $Y$, where $Y=B_{i Y} \times Z$ by the Sandwich lemma. Since $Z(x)$ is convex, we have $B_{j Y} \subseteq Z$, hence $B_{i Y}$ is perpendicular to $B_{j Y}$. On the other hand, since any geodesic in $X$ is parallel to an image flat, which is contained the span of $B_{1}$ through $B_{k}$, so $Y$ is spanned by those $B_{i Y}, 1 \leq i \leq k$.

Note that if $X_{i}^{*}$ is of rank 1 , then $S_{i}^{*}$, hence $S_{i}$ is connected, so either $S_{i} \subseteq$ $\mathbb{R}^{s}(\infty)$ or $S_{i} \subseteq Y(\infty)$. In this case $B_{i}$ is entirely in $\mathbb{R}^{s}$ or $Y$.

Write $X=\mathbb{R}^{s} \times B_{1 Y} \times \cdots \times B_{k Y}$. Each $\gamma \in \Gamma$ can be written in the form $\gamma=\left(\gamma_{0}, \gamma_{1 Y}, \ldots, \gamma_{k Y}\right)=\left(\gamma_{0}, \gamma_{Y}\right)$, since it preserves all the foliations $B_{i}$ and 
respects the product structure $X=\mathbb{R}^{s} \times Y$. Denote by $\Gamma_{Y}$ the group of all such $\gamma_{Y}$. Set $\widetilde{\Gamma^{*}}=\Gamma_{1}^{*} \times \cdots \times \Gamma_{k}^{*}$, where $\Gamma_{i}^{*}$ is the projection of $\Gamma^{*}$ onto the irreducible factor $X_{i}^{*}$. The group $\widetilde{\Gamma^{*}}$ is dense in $I_{0}\left(X^{*}\right)$.

We want to construct a surjective, continuous map $\tilde{f}: X^{*} \rightarrow Y$ which is $\left(\widetilde{\Gamma^{*}}, \Gamma_{Y}\right)$-equivariant. Then $\Gamma_{Y}$ will have a dense orbit in $Y$. Because $Y$ is locally compact, any sequence of isometries $\left\{\phi_{n}\right\}$ on $Y$ such that $\phi_{n}(y)$ converges for some $y \in Y$ will have a subsequence that converges to an element in $I(Y)$. Therefore, $I(Y)$ acts transitively on $Y$. Hence, it is a Lie group by the theorem of Montgomery and Zippin [20]. Thus, $I(X)$ is also a Lie group.

If $Q$ is a bounded subset in a Hadamard space $Y$, we will denote by $\tau_{Q}$ the infimum of the radii of the closed balls containing $Q$. There exists an unique point $y \in Y$ such that $Q \subseteq B_{\tau_{Q}}(y)$. This point is called the circumcenter of $Q$ and is denoted by $\star(Q)$. We refer the reader to page 26 of [1] for more details.

Lemma 13 Suppose $P$ and $Q$ are two bounded subsets in a Hadamard space $Y$ with Hausdorff distance $H d(P, Q)=h$. Then their circumcenters satisfy

$$
d(\star(P), \star(Q)) \leq \sqrt{h\left(\tau_{P}+\tau_{Q}+h\right)} .
$$

Proof Write $x=\star(P)$ and $y=\star(Q)$. Then we have $P \subseteq B_{\tau_{P}}(x) \cap B_{\tau_{Q}+h}(y)$. Let $z$ be the mid point of the geodesic segment from $x$ to $y$. Then since $Y$ is Hadamard, any $p \in P$ will satisfy

$$
d^{2}(p, z) \leq \frac{1}{2} d^{2}(p, x)+\frac{1}{2} d^{2}(p, y)-\frac{1}{4} d^{2}(x, y) \leq \frac{1}{2} \tau_{P}^{2}+\frac{1}{2}\left(\tau_{Q}+h\right)^{2}-\frac{1}{4} d^{2}(x, y) .
$$

Since this is true for any $p \in P$, the far right hand side of the above inequality must be bigger than $\tau_{P}^{2}$, hence

$$
d^{2}(x, y)<2 \tau_{Q}^{2}-2 \tau_{P}^{2}+4 \tau_{Q} h+2 h^{2} .
$$

Add this with the similar inequality obtained by reversing the role of $P$ and $Q$, we get

$$
d^{2}(x, y)<2 h\left(\tau_{P}+\tau_{Q}+h\right) .
$$

If one replace $z$ by the points on the geodesic segment from $x$ to $y$ that are very closed to $x$ or $y$, then the coefficient 2 in the right hand side can be removed.

We are now ready to construct $\tilde{f}$. Let $\pi$ be the projection map from $X$ onto $B_{1 Y}$. For any $x^{*} \in X_{1}^{*}$, write $Q^{*}\left(x^{*}\right)=\left\{x^{*}\right\} \times X_{2}^{*} \times \cdots \times X_{k}^{*}$, and $Q\left(x^{*}\right)=$ $\pi f\left(Q^{*}\left(x^{*}\right)\right)$. Define $\widetilde{f}_{1}\left(x^{*}\right)=\star\left(Q\left(x^{*}\right)\right)$ to be the circumcenter of $Q\left(x^{*}\right)$. For 
this we need to show that $Q\left(x^{*}\right)$ is always a bounded subset of $B_{1 Y}$. Fix a point $p^{*} \in Q^{*}$. For any $q^{*} \in Q^{*}$, there exists a $r$-flat $F^{*} \subseteq X^{*}$ containing both $p^{*}$ and $q^{*}$. So, if $r_{1}$ denotes the rank of $X_{1}^{*}$, then there are $m \leq r-r_{1}$ maximally singular geodesics in $F^{*}$, denoted by $l_{1}^{*}, \ldots, l_{m}^{*}$, each is perpendicular to $X_{1}^{*}$, $p^{*} \in l_{1}^{*}, q^{*} \in l_{m}^{*}$, and $l_{i}^{*} \cap l_{i+1}^{*} \neq \phi$ for $1 \leq i \leq m-1$. For each $l_{i}^{*}$, by Theorem $\mathrm{C}$, there exists geodesic $l_{i}$ in $X$ perpendicular to $B_{1 Y}$, such that the Hausdorff distance between $l_{i}$ and $f\left(l_{i}^{*}\right)$ is bounded by an uniform constant $R_{1}$. Therefore $d\left(\pi f\left(q^{*}\right), \pi f\left(p^{*}\right)\right) \leq 2 m R_{1} \leq 2 r R_{1}$, and $\tau_{Q} \leq 2 r R_{1}$. Since $f$ is uniformly continuous, and $H d\left(Q\left(x^{*}\right), Q\left(y^{*}\right)\right) \leq d\left(x^{*}, y^{*}\right)$, so Lemma 6 implies that $\widetilde{f}_{1}: X_{1}^{*} \rightarrow B_{1 Y}$ is continuous. It is also clear that for any $\gamma \in \Gamma$, we have $\widetilde{f}_{1}\left(\gamma_{1}^{*}\left(x^{*}\right)\right)=\gamma_{1 Y}\left(\widetilde{f}_{1}\left(x^{*}\right)\right)$. Now if we define $\widetilde{f}_{i}: X_{i}^{*} \rightarrow B_{i Y}$ similarly for each $1 \leq i \leq k$ and then take the product, we get a continuous map $\tilde{f}: X^{*} \rightarrow Y$ which is $\left(\widetilde{\Gamma^{*}}, \Gamma_{Y}\right)$-equivariant, where $\widetilde{\Gamma^{*}}=\Gamma_{1}^{*} \times \cdots \times \Gamma_{k}^{*}$. This implies the following.

Lemma 14 The map $\tilde{f}: X^{*} \rightarrow Y$ is surjective. Hence $\overline{\Gamma_{Y}}$ acts transitively on $Y$, and $I(X)$ is a Lie group.

Note that for any $x^{*} \in X^{*}$, we have $d\left(\tilde{f}\left(x^{*}\right), \pi_{Y} f\left(x^{*}\right)\right) \leq \sqrt{k} 2 r R_{1}$. So for any $y \in Y$,

$$
d(y, \tilde{f} \circ g(0, y)) \leq A+2 r \sqrt{k} R_{1} .
$$

Therefore $\tilde{f}$ is surjective by the following lemma.

Lemma 15 If a Hadamard space $Y$ is a topological manifold and if $h: Y \rightarrow Y$ is a continuous map within bounded distance from the identity map, then $h$ is surjective.

Proof The geodesic segment from $h(y)$ to $y$ gives a proper homotopy between $h$ and the identity map. Hence, $h$ induces the identity map on the top dimensional cohomology group with compact support, $H_{c}^{n}(Y), n=\operatorname{dim}(Y)$. The result follows.

\subsection{Proof of the Rigidity Theorem}

Now we can apply the techniques in [10], [11], [12] and [4] to finish the proof of Theorem B. From the previous subsection, $I(X)$ is Lie group. This implies that $X$ has no Euclidean factor, that is, we have the following lemma.

Lemma $16 X=Y=B_{1} \times \cdots \times B_{k}$. 
Proof First, notice that $\Gamma$ does not contain any Clifford translations. This is because the set of Clifford translations in $\Gamma$ forms a normal abelian subgroup. By the Main Theorem of [11], $\Gamma^{*}$ does not contain any nontrivial normal abelian subgroup, since $X^{*}$ has no Euclidean de Rham factor.

Now let us assume that $X=\mathbb{R}^{s} \times Y$ for $s>0$. We want to derive a contradiction. Note that $Y$ is not a point, for otherwise $\Gamma$ would contain a translation by the Bieberbach Theorem.

Denote by $\Gamma_{\mathbb{R}}$ and $\Gamma_{Y}$ the projection of $\Gamma$ on $\mathbb{R}^{s}$ and $Y$, respectively. Since $I(X)$ is a Lie group, the proof of Lemma $\mathrm{A}$ in [11] says that $\Gamma_{Y}$ is discrete. Let $N$ be the kernel of $\Gamma \rightarrow \Gamma_{Y}$. The proof of Theorem 4.1 of [12] says that either $\Gamma_{\mathbb{R}}$ is discrete or $N$ contains a Clifford translation. Since $\Gamma^{*}$ is assumed to be irreducible, $\Gamma_{\mathbb{R}}$ can not be discrete. Since $\Gamma$ contain no Clifford translation, so does the subgroup $N$. This completes the proof. (Note that in this argument, Proposition 2.3 of [4] is used. But it is obviously valid in the singular case.)

In our next argument we also need an extension of Theorem 2.4 of [4] to singular spaces. The proof there is based on four lemmas. We already established singular version of Lemma $2.4 \mathrm{a}$ - see Lemma 4 . The same sort of modification works for Lemma 2.4c. The proof of Lemma $2.4 \mathrm{~b}$ holds without change. For the proof of Theorem 2.4 the following replacement of Lemma $2.4 \mathrm{~d}$ suffices.

Lemma 17 Let $A \subseteq I(X)$ be a nontrivial abelian subgroup such that normalizer $D$ of $A$ in $I(X)$ satisfies the duality condition. Then $I(X)$ contains a Clifford translation.

Proof (cf proof of Lemma 2.4d of [4]) Let $L(A) \subseteq X(\infty)$ denote the limit set of $A$. By Lemma 2.4b of [4], $L(A)$ is not empty. Let $x \in L(A)$, then by Lemma 2.4c there exist a unique point $y \in L(A)$ such that $x$ can be joined to $y$. If $z \in H(\infty)$ is a point that can be joined to $y$ then by Lemma 4 $z \in \overline{D(x)} \subseteq L(A)$ and therefore $z=x$. So $X$ admits Clifford translations along the geodesics joining $x$ to $y$ by Sandwich Lemma.

Denote by $\Gamma_{i}=\Gamma_{i Y}$ the projection of $\Gamma$ on $B_{i}$, and write $G_{i}=\left(\overline{\Gamma_{i}}\right)_{0} \subseteq I_{0}\left(B_{i}\right)$ the identity component of its closure. By the proof of Lemma 3.1 of [10], which essentially uses Theorem 2.4 of [4] (cf Lemma 17), we know that each $G_{i}$ is a centerless semisimple Lie group of noncompact type. Fix a maximal compact subgroup $K \subseteq G_{i}$. By taking the circumcenter of an orbit, we know that $K$ will fix some point in $B_{i}$. Let $p \in B_{i}$ be a fixed point of $K$. Since $B_{i}$ is locally compact, the isotropy subgroup of $G_{i}$ at $p$ is compact, and equals to $K$ since 
$K$ is maximal compact. So the transitivity of the action of $G_{i}$ on $B_{i}$ gives a homeomorphism between $B_{i}$ and $G_{i} / K$. The metric $d$ on $B_{i}$ is a $G_{i}$-invariant Hadamard metric. By the following lemma, $d$ must be smooth, so $\left(B_{i}, d\right)$ is a global Riemannian symmetric space of noncompact type. Therefore $(X, d)$ is symmetric, and isometric to $\left(X^{*}, d^{* *}\right)$ by the theorem of Mostow [19]. This completes the proof of Theorem B.

Lemma 18 Suppose $G$ is a semisimple Lie group of noncompact type and that $K$ a maximal compact subgroup. If $d$ is a Hadamard metric on $G / K$ that is $G$-invariant, then $(G / K, d)$ is Riemannian symmetric space of noncompact type.

Proof If $G$ is not simple, we may take a lattice $\Gamma \subseteq G$ and consider $X^{*}=G / K$ equipped with $d^{*}$ symmetric and $X=G / K$ equipped with $d$. By the previous arguments, we know that $(X, d)$ will be a product space of $\left(G_{i} / K_{i}, d_{i}\right)$, where those $G_{i}$ are the simple factors of $G$. Therefore we may assume that $G$ is simple.

First consider the case when the rank of $G$ is 1 . In this case, the geodesic sphere in $d$ is a single $K$ orbit, so it coincides with a geodesic sphere with the same center under $d^{*}$, the symmetric metric. For any two points $p, q \in X$, let $m$ be the midpoint under the metric $d$. Then there are two geodesic spheres under $d^{*}$, centered at $p$ and $q$, respectively, so that $m$ is their unique intersection point. This implies that $m$ must be on the geodesic segment from $p$ to $q$ with respect to the metric $d^{*}$. So the geodesics in $d$ and $d^{*}$ have the same images.

Fix $p$ and $q$ with $d(p, q)=1$. Let $d^{*}$ be the symmetric metric on $X=G / K$ such that $d^{*}(p, q)=1$. Let $\varphi_{t}$ be a one parameter subgroup of $G$ such that $\gamma(t)=\varphi_{t}(p)$ is the unit speed geodesic under $d^{*}$ with $\gamma(1)=q$.

By considering $\varphi_{\frac{1}{2^{n}}}$, we know that $d(\gamma(0), \gamma(t))=d^{*}(\gamma(0), \gamma(t))$ for any rational number $t$, whose denominator is a power of two. Hence, it also holds for any $t \in \mathbb{R}$ by continuity. For any $p^{\prime}, q^{\prime} \in X$, and for any $\varphi \in G$ such that $\varphi\left(p^{\prime}\right)=$ $\gamma(0)$ and such that $\varphi\left(q^{\prime}\right)$ lies in the image of $\gamma$, we have $d\left(p^{\prime}, q^{\prime}\right)=d^{*}\left(p^{\prime}, q^{\prime}\right)$. So $d$ is symmetric in this case.

Now assume $G$ is simple with rank $r \geq 2$. We need the following.

Sublemma Suppose $G$ is a connected, centerless, semisimple Lie group of noncompact type, and $K$ a maximal compact subgroup. Let $\mathfrak{g}=\mathfrak{k}+\mathfrak{p}$ be the Cartan decomposition of the Lie algebras. Suppose $\mathfrak{a}$ is a maximal abelian subalgebra in $\mathfrak{p}$, and $A=\exp (\mathfrak{a})$. Denote by $M=Z_{K}(A)$ the centralizer of $A$ 
in $K$, and denote by $Y$ the fixed point set in $G / K$ of $M$. Then $Y$ is a totally geodesic submanifold in $G / K$ containing the maximal flat $F=A x_{0}$, where $x_{0}=K$. Denote by $r$ the rank of $G / K$.

(1) $M=\{1\}$ when and only when $G / K$ is the product of $r$ copies of the hyperbolic plane $\mathbb{H}^{2}$.

(2) If $G$ is simple, then either $Y=\left(\mathbb{H}^{2}\right)^{r}$, or $Y=F \cong \mathbb{R}^{r}$, depending on whether $G / K$ is Hermitian symmetric or not.

Now let us use Sublemma to finish the proof of Lemma 18. Let $F \subseteq B=G / K$ be a maximal flat under $d^{*}$, with $K$ the isotropy subgroup at $x_{0} \in F$. First we claim that $F$ is $d$-convex.

Let $\mathfrak{g}=\mathfrak{k}+\mathfrak{p}$ be the Cartan decomposition of the Lie algebras of $G$ and $K$, and $\mathfrak{h}$ a maximal abelian subalgebra contained in $\mathfrak{p}$. Write $A=\operatorname{exph}$ so that $F=A x_{0}$. As in Sublemma, denote by $M$ the centralizer of $A$ in $K$, and denote by $Y$ the fixed point set of $M$ in $B$. Then $Y \subseteq B$ is totally geodesic in $B$ under $d^{*}$.

Note that $Y=G_{1} / K_{1}$ is a symmetric space of nonpositive curvature, with $G_{1} \subseteq G$ and $K_{1} \subseteq K$. Since $Y$ is the fixed point set of $d$-isometries, so it is $d$-convex, and $G_{1}$ acting transitively on $Y$ as both $d^{*}$ and $d$-isometries.

By Sublemma, either $Y=F$, in which case $F$ is a $d$-flat, or $Y=\left(\mathbb{H}^{2}\right)^{r}$. In this case, the arguments in the previous subsections implies that $(Y, d)$ must split as the product of $r$ surfaces, each is symmetric since $\left(\mathbb{H}^{2}, d^{*}\right)$ has rank 1 . So $d$ is a symmetric metric on $Y$, and $F$ is a $d$-flat.

Now consider any maximal flat $F \subseteq B$ passing through $x_{0}$. The restriction of $d$ to each flat $F$ is a Euclidean metric which is invariant under the Weyl group, which acts irreducibly on $F$ since $G$ is assumed to be simple. So $\left.d\right|_{F}=$ $\left.\lambda(F) d^{*}\right|_{F}$. Since any two flats through $x_{0}$ can be joined by finitely many flats through $x_{0}$ so that each one intersects the next at more than one point, $\lambda(F)$ must be constant, and $d=\lambda d^{*}$ is symmetric. This completes the proof of Lemma 18 assuming Sublemma.

Proof of Sublemma Let us start with part (1). Without loss of generality, we may assume that $G$ is simple. Assume $M=1$, we want to conclude that $G / K$ is the hyperbolic plane. Let $r$ be the rank of $G / K$.

Since the Lie algebra $\mathfrak{m}$ of $M$ is trivial, $\mathfrak{h}_{\mathbb{C}}$ is a Cartan subalgebra for $\mathfrak{g}_{\mathbb{C}}$, the complexification of $\mathfrak{g}$. Denote by $G^{\prime}$ the real points of $G_{\mathbb{C}}$ under the conjugation 
of $\mathfrak{g}$ in $\mathfrak{g}_{\mathbb{C}}$. Then $G=\operatorname{Ad}(G)$ is the identity component of $G^{\prime}$. Denote by $K^{\prime}$ the maximal compact subgroup of $G^{\prime}$ with identity component $K$.

Let $P \cong\left(\mathbb{Z}_{2}\right)^{r}$ be the group of 2 -torsion elements in $H_{\mathbb{C}}=\exp \left(\mathfrak{h}_{\mathbb{C}}\right)$. Then $P$ is contained in $Z_{K^{\prime}}(A)$, the centralizer of $A$ in $K^{\prime}$.

Note that the number of connected components in $G^{\prime}$ is bounded by the order of the outer automorphism group $Q=\operatorname{Aut}(G) / \operatorname{Int}(G)$. So the assumption $M=Z_{K}(A)=1$ implies that $2^{r} \leq|Q|$.

By the Table 10 on page 156 of [18], we see that this is impossible unless $\mathfrak{g}_{\mathbb{C}}$ is $\mathfrak{a}_{1}=\mathfrak{s l}(2, \mathbb{C})$ or $\mathfrak{d}_{4}=\mathfrak{s o}(8, \mathbb{C})$. In the $\mathfrak{d}_{4}$ case, in fact in all four classical Lie algebra cases, it can be easily checked that $M \neq 1$ except for $\mathfrak{a}_{1}$. This completes the proof of part (1).

For part (2), let us apply part (1) to the symmetric space (which may contain a Euclidean factor now) $Y$, since $M_{1}=Z_{K_{1}}(A)$ is trivial, by part (1), we know that $Y=\left(\mathbb{H}^{2}\right)^{s} \times \mathbb{R}^{r-s}$. We want to show that $s$ is either 0 or $r$ if $G$ is simple.

Let $M^{\prime}=N_{K}(A)$ be the normalizer of $A$ in $K$. Then $M^{\prime}$ ( or $M$ ) consists of elements in $K$ that stabilize $F$ ( fix every point in $F$ ). $M$ is a normal subgroup of finite index in $M^{\prime}$, and $W=M^{\prime} / M$ is Weyl group which acts irreducibly on $F$. For each $g \in M^{\prime}$, since $g M g^{-1}=M$, we have $g(Y)=Y$. Now since any isometry of $Y$ preserves the Euclidean factor, we know that the $\mathbb{R}^{r-s}$ part of $F$ is invariant under $W$. So either $s=0$ or $s=r$. This completes the proof of Sublemma.

Remark Note that the CAT(0) assumption on $d$ in Lemma 18 is necessary. When rank $r \geq 2$, there are $G$-invariant length metric on $G / K$ which are geodesic. The simplest example would be taking product of two copies of symmetric spaces, and let $d=d_{1}+d_{2}$ where $d_{i}$ are the standard metrics on the factors. Then clearly $d$ is $G$-invariant but not Riemannian. More generally, Planche showed [21] that the set of $G$-invariant Finsler metrics on $G / K$ is in one-to-one correspondence with the set of $W$-invariant norms on a maximal flat $F$. (Here $W$ denotes the Weyl group.) Some of these metrics are even uniquely geodesic, that is, any two points in $G / K$ can be joined by a unique geodesic segment. 


\section{References}

[1] W Ballmann, Lectures on Spaces of Nonpositive Curvature, Birkhäuser (1995)

[2] W Ballmann, M Gromov, V Schroeder, Manifolds of Nonpositive Curvature, Birkhäuser (1985)

[3] M Bridson, A Haefliger, Spaces of nonpositive curvature, Springer-Verlag, Berlin, Heidelberg, New York (1999)

[4] S-S Chen, P Eberlein, Isometry groups of simply connected manifolds of nonpositive curvature, Illinois Journal of Math. 24 (1980) 73-103

[5] R Charney, MW Davis, Singular metrics of nonpositive curvature on branched covers of Riemannian manifolds, Amer. J. Math. 115 (1993) 929-1009

[6] R Charney, M W Davis, The polar dual of a convex polyhedral set in hyperbolic space, Michigan Math. J. 42 (1995) 479-510

[7] R Charney, M W Davis, G Moussong, Nonpositively curved piecewise Euclidean structures on hyperbolic manifolds, Michigan Math. J. 44 (1997) 201-208

[8] M W Davis, T Januszkiewicz, Hyperbolization of polyhedra, J. Diff. Geom. 34 (1991) 347-388

[9] $\mathbf{P}$ Eberlein, Rigidity of lattices of nonpositive curvature, Ergodic Theory \& Dynamical Systems, 3 (1983) 47-85

[10] P Eberlein, Isometry groups of simply connected manifolds of non-positive curvature, II, Acta Math. 149 (1982) 41-69

[11] P Eberlein, Euclidean de Rham factor of a lattice of nonpositive curvature, J. Diff. Geometry, 18 (1983) 209-220

[12] P Eberlein, Lattices in spaces of nonpositive curvature, Ann. Math. 111 (1980) 435-476

[13] A Eskin, B Farb, Quasi-flats and rigidity in higher rank symmetric spaces, J. Amer. Math. Soc. 10 (1997) 653-692

[14] M Gromov, Hyperbolic groups, Essays in group theory (S M Gersten, ed.), MSRI Publ. 8, Springer-Verlag, Berlin, Heidelberg, New York (1987) 75-264

[15] M Gromov, W Thurston, Pinching constants for hyperbolic manifolds, Invent. Math. 89 (1987) 1-12

[16] B Kleiner, B Leeb, Rigidity of quasi-isometries for symmetric spaces and Euclidean buildings, Inst. Hautes Études Sci. Publ. Math. No. 86 (1997) 115197

[17] B Leeb, A characterization of irreducible symmetric spaces and Euclidean buildings of higher rank by their asymptotic geometry, preprint (1997)

[18] O Loos, Symmetric spaces: Volume II, W.A. Benjamin, Inc. (1969)

[19] G Mostow, Strong rigidity of locally symmetric spaces, Annals of Math. Studies 78, Princeton University Press (1973) 
[20] D Montgomery, L Zippin, Topological Transformation Groups, Interscience, New York (1955)

[21] P Planche, Structures de Finsler invariantes sur les espaces symétriques, CR. Acad. Sci. Paris, 321 (1995) 1455-1458

[22] M S Raghunathan, Discrete subgroups of Lie groups, Springer-Verlag, Berlin, Heidelberg, New York (1972)

[23] A Selberg, Recent developments in the theory of discontinuous groups of motion of symmetric spaces, Springer Lecture Notes in Math. 118, Springer-Verlag, Berlin, Heidelberg, New York (1969) 